\begin{tabular}{|c|l|}
\hline Title & Orbital and spin polarizations induced by current through a helical atomic chain \\
\hline Author(s) & Otsuto, Ryosuke; Y atabe, Y uya; A kera, Hiroshi \\
\hline Citation & $\begin{array}{l}\text { Physical Review B, 104/3), 035431 } \\
\text { https://doi.org/10.1103/PhysRevB.104.035431 }\end{array}$ \\
\hline Issue Date & 2021-07-27 \\
\hline Doc URL & http://hdl.handle.net/2115/82580 \\
\hline Rights & Copyright (2021) by The A merican Physical Society. \\
\hline Type & article \\
\hline File Information & PhysRevB.104.035431.pdf \\
\hline
\end{tabular}

Instructions for use 


\title{
Orbital and spin polarizations induced by current through a helical atomic chain
}

\author{
Ryosuke Otsuto, Yuya Yatabe $\odot$, and Hiroshi Akera $\odot$ \\ Division of Applied Physics, Graduate School and Faculty of Engineering, Hokkaido University, Sapporo, Hokkaido 060-8628, Japan
}

(Received 25 March 2021; revised 23 May 2021; accepted 14 July 2021; published 27 July 2021)

\begin{abstract}
Current-induced spin and orbital polarizations are theoretically studied in a helical atomic chain with $p_{x}, p_{y}$, and $p_{z}$ orbitals in each atom in the linear response to the chemical-potential difference between source and drain electrodes. It is shown that these polarizations are parallel to the helix axis and proportional to the curvature of the cylinder surface defined by the helix in the small curvature region. This proportionality suggests that the curvature generates the coupling between the current and the atomic orbital angular momentum and therefore influences the magnitude of induced spin polarization through the $L S$ coupling. It is also found that current-induced polarizations are nonzero even when the pitch is enforced to vanish. This suggests the possibility that nonchiral structures with curvature can produce current-induced polarizations. With this possibility in mind, it is proposed that the chirality in the current, rather than that in the structure, determines the existence of such current-induced polarizations.
\end{abstract}

DOI: 10.1103/PhysRevB.104.035431

\section{INTRODUCTION}

Spintronics [1] utilizes the electron spin, in addition to the charge, for information processing. Extensive studies have been performed to create the spin polarization required in nonmagnetic systems in the form of crystals and fabricated microstructures using the spin-orbit interaction (SOI). Recently, studies have been extended to organic molecules [2]. A device formed by a single organic molecule, when realized, will achieve ultimate miniaturization. One of the challenges faced by organic spintronics is small SOI: the SOI is small in light-element atoms, such as carbon and oxygen, which form organic molecules. Therefore, the efficiency of generating spin polarization in organic molecules was previously thought to be low.

Contrary to such a preconception, a high efficiency of spin filtering, comparable to the highest efficiency in other systems, has been discovered in helical organic molecules such as double-stranded DNA, oligopeptides, and helicene [3-13] (also recently found in supramolecular helical nanofibers [14] and superhelical polyaniline microfibers [15]) and named the chirality-induced spin selectivity (CISS; see, for a review, [16]). Such high-efficiency spin filtering has been observed in photoelectron transmission through a self-assembled monolayer of organic molecules $[4,6,7,10]$ as well as in electron transport through a single organic molecule [5,7-9,11-14]. In this paper we focus on the spin selectivity in electron transport in view of recent progress in its application to a magnetic memory device [17] and a light-driven reconfigurable spin filter [18]. The high spin selectivity in spite of the small SOI in organic molecules suggests an undiscovered mechanism of efficiency enhancement by the helical geometry, which might also be useful as a guiding principle in designing a high-efficiency spin filter in other systems.

Experimental findings which suggest the mechanism of the spin selectivity in helical organic molecules are as follows: (a) the spin selectivity reverses its sign with changing chirality [8-11], (b) it increases with the number of monomers in the organic molecule $[4,5,7,12]$ and decreases by compression along the length via the applied force [19], and (c) its magnitude depends strongly on the monomer type, that is, whether it is a base [in single-stranded DNA (ssDNA)] $[3,4]$ or amino acid (in oligopeptide) [7,9]. A number of calculations [20-32] have been made on transport through a single model molecule consisting of sites, each of which has a single orbital. Each site in the model represents each monomer of helical organic molecules [21,22,24-32], each atom of helicene [23], or each site in an effective tight-binding model [20]. Since the SOI cannot be expressed by a single orbital within one site, it is incorporated in hopping integrals between sites in the model Hamiltonian. Such studies in the single-orbital model successfully reproduce some of the observed features, the chirality dependence $[22,23,26,27]$, and the increase with the number of monomers $[21,22,25]$. However, studying the geometry dependence of the spin selectivity, such as observed in the experiment [19], with an aim to clarify the mechanism of the high efficiency originating from the helical geometry, needs to take into account intra-atomic SOI ( $L S$ coupling) in a model with more than one atomic orbital.

Several theoretical studies [33-39] have proposed a mechanism of the spin selectivity on the basis of intra-atomic SOI. These theories have introduced an additional feature in the Hamiltonian: orbital anisotropy giving the splitting of $p$ levels between $\pi$ and $\sigma$ orbitals [33-37] and that between the $p$ orbital tangential to the helix and others [38] and electron-hole pairing caused by many-body correlation [39]. It is interesting, however, to ask whether only the helical arrangement of atoms can produce any spin selectivity in a model with isotropic atoms connected by geometry-dependent hopping matrix elements. Numerical calculations for specific molecules have also been performed using the density 
functional method [40-45] and a tight-binding model [46], which will be necessary to obtain the spin-selectivity efficiency in a specific organic molecule. An alternative approach is still desirable in order to reach a common mechanism of the enhanced spin selectivity which has been observed in various helical molecules.

In this paper we theoretically study the dependence of the spin selectivity on the radius and the pitch of the helix in the simplest helical structure, that is, a helical atomic chain consisting of a single element, with an aim to extract a common atomic mechanism which arises from the helical geometry. We take into account $p_{x}, p_{y}$, and $p_{z}$ orbitals in each atom in order to incorporate the intra-atomic SOI. Recent experiments [47-51] have actually observed a helical atomic chain formed in a single-walled carbon nanotube by iodine atoms $[47,48]$ and tellurium atoms [50], in a double-walled carbon nanotube by selenium atoms [49], and in a boron nitride nanotube by tellurium atoms [51]. Electronic band structure calculations of helical atomic chains have also been performed [49-53], while effects of the SOI in the electron transport remain to be studied. In studying the electron transport through a helical atomic chain we neglect the coupling to the nanotube. Such a decoupled-chain model [53] is expected to describe well the transport through an atomic chain in an insulating boron nitride nanotube [51]. On the other hand, the transport through a conductive carbon nanotube in the helicoidal electric field created by wrapping the nanotube with less conductive ssDNA was theoretically predicted to exhibit a high spin selectivity [54] and has subsequently been experimentally confirmed [55] (for a recent theory, see [56]).

In this paper we calculate the current-induced spin polarization (CISP) in our model of a helical atomic chain. The CISP has been extensively studied theoretically [57-59] and experimentally [60-63] in various systems, and the existence of the CISP in a helical structure has been theoretically shown in a model with the SOI in intersite hopping integrals [64] and, recently, in a continuum model for the CISS [65]. The reason why we calculate the CISP is that we consider the CISP to be fundamental for the spin selectivity. In fact the CISP is known to produce the spin diffusion current through an attached conductor, as demonstrated experimentally [66-68]. Recently, the CISP in a chiral inorganic crystal was detected by the spin diffusion current [69]. In addition to the CISP, we study the polarization of the atomic orbital angular momentum, called the current-induced orbital polarization (CIOP), which acts on the spin as an effective magnetic field through the $L S$ coupling. The CIOP was recently studied in crystals [70-73], and the orbital current was also calculated in the orbital Hall effect in crystals [74-77] and in the orbital filter in a model of CISS [78]. Here we calculate both CIOP and CISP as a function of the radius and the pitch of the helix in the linear response to the chemical-potential difference between source and drain electrodes in the Landauer approach.

The organization of the paper is as follows. Section II describes the model and basis vectors for a helical atomic chain as well as the Landauer approach for calculating the CIOP and CISP. Section III presents calculated results of the energy eigenvalue and expectation values in each eigenstate of orbital and spin angular momenta as well as their current-induced polarizations. Section IV gives conclusions.

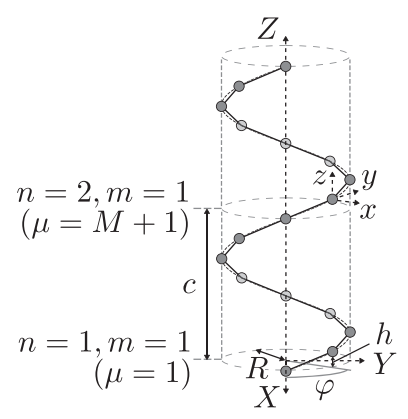

FIG. 1. Helical atomic chain with radius $R$ and pitch $c$. The unit cell contains $M$ atoms. Here $M=8 . h=c / M$, and $\varphi=2 \pi / M . n$ (= $1,2, \ldots, N)$ labels the unit cell, $m(=1,2, \ldots, M)$ labels each atom in the unit cell, and $\mu=M(n-1)+m$.

\section{MODEL AND METHOD}

\section{A. Model of a helical atomic chain}

Our system is a chain of atoms equally spaced along the helix. In this paper we restrict our discussion to the atomic arrangement which has an integer number $M$ of atoms in one turn of the helix as in the previous study in [25]. Owing to this restriction, the system has symmetry with respect to the translation along the helix axis by the pitch $c, \hat{T}(c)$, and the corresponding unit cell contains $M$ atoms. Then we denote the position of each atom by two integers $(n, m): n$ (= $1,2, \ldots, N)$ labels the unit cell and $m(=1,2, \ldots, M)$ labels each atom in the unit cell, as shown in Fig. 1. We impose the periodic boundary condition with a periodicity of $N$ unit cells.

We employ the simplest tight-binding model which can describe the dependence of the spin selectivity on the geometry of the helix based on intra-atomic SOI. Thus, we consider only $p_{x}, p_{y}$, and $p_{z}$ orbitals in each atom. We describe the electron transport along the helix by nearest-neighbor hopping integrals, which we express in terms of the Slater-Koster parameters, $V_{\mathrm{pp} \sigma}$ and $V_{\mathrm{pp} \pi}$, for $\sigma$ and $\pi$ bondings [79].

\section{B. Basis vectors}

Here we introduce the $x y z$ coordinate system for each atom in addition to the $X Y Z$ coordinate system fixed to the helix. As shown in Fig. 1, both $z$ and $Z$ axes are taken along the helix axis, while the $x$ and $y$ axes for the atom at $(n, m)$ are obtained by rotating the $X$ and $Y$ axes around $Z$ by $(m-1) \varphi$, with $\varphi=$ $2 \pi / M$, so that the $x$ axis is perpendicular to the cylindrical surface containing the helix.

We construct basis vectors by taking into account, in addition to the translational symmetry along the helix axis, the symmetry with respect to the screw operation $\hat{S}_{c}(\varphi)$, consisting of the rotation around $Z$ by $\varphi$ and the translation along $Z$ by $h=c / M$. With the screw operation of atomic orbitals in mind, we express each basis vector in a linear combination of atomic orbitals defined in the $x y z$ coordinate system, $\left|p_{\alpha} ; n m\right\rangle(\alpha=x, y, z)$, in which $p_{x}$ and $p_{y}$ are obtained by rotating $p_{X}$ and $p_{Y}$ around $Z$ by $(m-1) \varphi$ for the atom at $(n, m)$. Since the SOI couples the spin to the orbital, we also introduce spin vectors defined in the $x y z$ coordinate system [80], $|\sigma ; m\rangle$, which are vectors obtained by rotating $|\sigma ; 1\rangle$ 
around $Z$ by $(m-1) \varphi$ :

$$
|\sigma ; m\rangle=\exp \left[-i \hat{\sigma}_{z}(m-1) \varphi / 2\right]|\sigma ; 1\rangle,
$$

where $|\sigma ; 1\rangle$ is the vector defined in the $X Y Z$ coordinate system (at $m=1$, the $x$ and $y$ axes coincide with the $X$ and $Y$ axes, respectively), with $\sigma(= \pm 1)$ being the $Z$ component of spin. Then we write each basis vector in a linear combination of $\left|p_{\alpha} ; n m\right\rangle|\sigma ; m\rangle$ :

$$
\left|p_{\alpha} \sigma\right\rangle=\sum_{n, m} C_{n m}\left|p_{\alpha} ; n m\right\rangle|\sigma ; m\rangle .
$$

Since $[\hat{H}, \hat{T}(c)]=0$ and $\left[\hat{H}, \hat{S}_{c}(\varphi)\right]=0$, with $\hat{H}$ being the Hamiltonian, we impose $\hat{T}(c)\left|p_{\alpha} \sigma\right\rangle=e^{i k c}\left|p_{\alpha} \sigma\right\rangle$ and $\hat{S}_{c}(\varphi)\left|p_{\alpha} \sigma\right\rangle=e^{i J \varphi+i k h}\left|p_{\alpha} \sigma\right\rangle$, where $k$ is the linear momentum and $J$ is the angular momentum. Then we obtain the normalized basis vector in the following form:

$$
\left|p_{\alpha} \sigma k J\right\rangle=\sum_{n, m} C_{n m}^{k J}\left|p_{\alpha} ; n m\right\rangle|\sigma ; m\rangle,
$$

with

$$
C_{n m}^{k J}=(N M)^{-1 / 2} \exp \left(i k z_{n m}+i J \varphi m\right),
$$

where

$$
\begin{aligned}
z_{n m} & =n c+m h, \\
k & =2 \pi n_{k} /(N c), \\
n_{k} & =0,1, \ldots, N-1, \\
J & =m_{J}+\frac{1}{2}, \\
m_{J} & =0,1, \ldots, M-1 .
\end{aligned}
$$

Here we introduce a single integer, $\mu$, defined by

$$
\mu=M(n-1)+m,
$$

to identify the atom at

$$
z_{\mu}=\mu h+c=z_{n m},
$$

and a single quantum number defined by

$$
\kappa=k+(2 \pi / c) J
$$

with $0<k<2 \pi / c$ and $J-1 / 2=0,1, \ldots, M-1$. Then each basis vector is written in terms of $z_{\mu}$ and $\kappa$ :

$\left|p_{\alpha} \sigma \kappa\right\rangle=(N M)^{-1 / 2} \sum_{\mu} \exp \left(i \kappa z_{\mu}\right)\left|p_{\alpha} ; \mu\right\rangle|\sigma ; \mu\rangle=-\left|p_{\alpha} \sigma k J\right\rangle$,

with $\quad\left|p_{\alpha} ; \mu\right\rangle=\left|p_{\alpha} ; n m\right\rangle \quad$ and $\quad|\sigma ; \mu\rangle=$ $\exp \left(-i \hat{\sigma}_{z} \mu \varphi / 2\right)|\sigma ; 0\rangle=(-1)^{n-1}|\sigma ; m\rangle$.

Eigenvectors are written in a linear combination of basis vectors:

$$
|v \kappa\rangle=\sum_{\alpha \sigma} A_{\alpha \sigma}^{\nu \kappa}\left|p_{\alpha} \sigma \kappa\right\rangle, \quad v=1, \ldots, 6 .
$$

Each eigenvector $A_{\alpha \sigma}^{\nu \kappa}$ and the corresponding eigenvalue $\varepsilon_{\nu \kappa}$ are obtained by solving the following equation:

$$
\sum_{\beta \sigma^{\prime}}\left\langle p_{\alpha} \sigma \kappa|\hat{H}| p_{\beta} \sigma^{\prime} \kappa\right\rangle A_{\beta \sigma^{\prime}}^{\nu \kappa}=\varepsilon_{\nu \kappa} A_{\alpha \sigma}^{\nu \kappa}
$$

\section{Landauer approach for current-induced polarizations of orbital and spin angular momenta}

To both ends of the helical atomic chain we attach the source and drain electrodes, which have chemical potentials $\mu_{S}$ and $\mu_{D}$, respectively. We assume that eigenstates traveling in the direction from the source to the drain and those traveling in the opposite direction are occupied up to $\mu_{S}$ and $\mu_{D}$, respectively, while the remaining states are empty. In the linear response to the chemical-potential difference, that is, in the limit of $\Delta \mu \equiv\left|\mu_{S}-\mu_{D}\right| \rightarrow 0$, we obtain, for polarizations of orbital and spin angular momenta, $\left\langle L_{\gamma}\right\rangle$ and $\left\langle\sigma_{\gamma}\right\rangle$ $(\gamma=X, Y, Z)$,

$$
\left\langle L_{\gamma}\right\rangle=\sum_{\text {channel } \kappa}^{S \rightarrow D}\left\langle v_{\kappa} \kappa\left|\hat{L}_{\gamma}\right| v_{\kappa} \kappa\right\rangle \rho_{\kappa} N c \Delta \mu
$$

and

$$
\left\langle\sigma_{\gamma}\right\rangle=\sum_{\text {channel } \kappa}^{S \rightarrow D}\left\langle v_{\kappa} \kappa\left|\hat{\sigma}_{\gamma}\right| v_{\kappa} \kappa\right\rangle \rho_{\kappa} N c \Delta \mu,
$$

in which the sum is taken over all transport channels from the source to the drain and $\rho_{\kappa} N c \Delta \mu$ is the number of electrons in the energy window of $\Delta \mu$ in each channel. Here such channels are identified by the quantum number $\kappa$ at the Fermi energy. Then the band index and the group velocity of the channel $\kappa$ are denoted by $v_{\kappa}$ and $v_{\kappa}$, respectively. $\rho_{\kappa}=1 /\left(h v_{\kappa}\right)$ is the density of states of the channel $\kappa$ divided by $N c$, the length along the $Z$ axis of the helix. The operator $\hat{L}_{\gamma}$ is defined by

$$
\hat{L}_{\gamma}=\sum_{\mu \alpha \beta}\left|p_{\alpha} ; \mu\right\rangle\left\langle p_{\alpha} ; \mu\left|\hat{L}_{\gamma}^{\mu}\right| p_{\beta} ; \mu\right\rangle\left\langle p_{\beta} ; \mu\right|,
$$

where $\hat{L}_{\gamma}^{\mu}$ is the orbital angular momentum operator (divided by $\hbar$ ) in the $\mu$ th atom.

\section{CALCULATED RESULTS}

\section{A. Atomic parameters}

We use atomic parameters for iodine. We employ the energy separation between spin-orbit-split $p$ levels, $1.029 \mathrm{eV}$ [81], to determine the $L S$ coupling strength. We use values of the Slater-Koster parameters $V_{\mathrm{pp} \sigma}=2.282 \mathrm{eV}$ and $V_{\mathrm{pp} \pi}=$ $-0.549 \mathrm{eV}$, which have been obtained to reproduce energy bands in the density functional calculation for the interatomic distance $d=2.9 \AA[53]$.

\section{B. Energy eigenvalue and expectation values of orbital and spin angular momenta}

Figure 2(a) presents the energy of eigenstate $v \kappa, \varepsilon_{\nu \kappa}$, as a function of $\kappa$ for $v=1, \ldots, 6$. The spin splitting due to the broken inversion symmetry in a helical atomic chain appears between the spin-up state at $\kappa-\pi / c$ and the spindown state at $\kappa+\pi / c$. The reason is that, for the quantum number $\kappa$ which includes the spin angular momentum, the spin-degenerate states in the absence of the SOI are the spinup state at $\kappa-\pi / c$ and the spin-down state at $\kappa+\pi / c$ [80].

The energy band structure of helical chains can be understood by starting with that of the straight chain $(R \rightarrow \infty)$ in the absence of the SOI which consists of $\sigma$ bands formed 

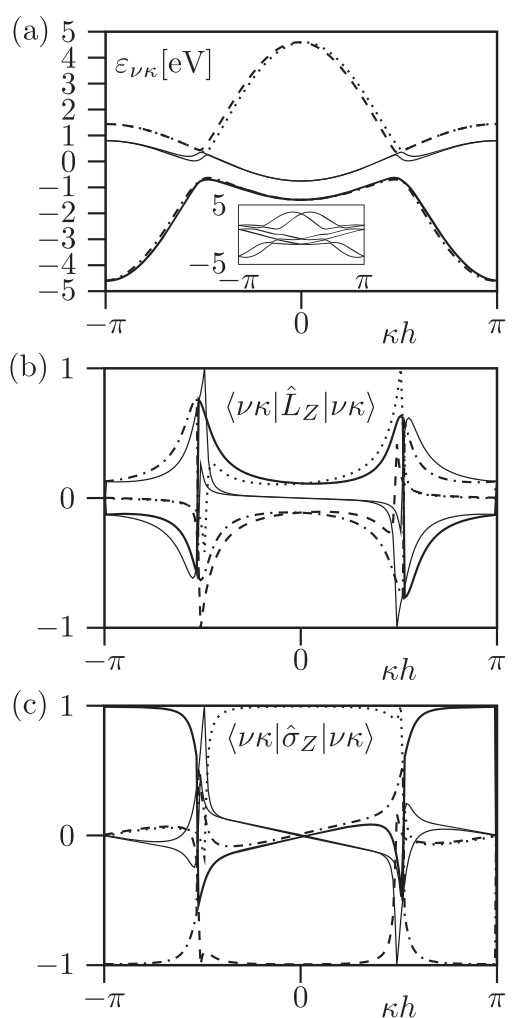

FIG. 2. Eigenstates in a helical atomic chain having $M=100$ and $h=0.1 \AA$, which gives $c=10 \AA$ and $R \approx 46.14 \AA$. (a) Eigenen$\operatorname{ergy} \varepsilon_{\nu \kappa}$, (b) the expectation value of the orbital angular momentum $\left\langle\nu \kappa\left|\hat{L}_{Z}\right| \nu \kappa\right\rangle$, and (c) that of the spin angular momentum $\left\langle\nu \kappa\left|\hat{\sigma}_{Z}\right| \nu \kappa\right\rangle$, plotted as a function of the quantum number $\kappa$ for six bands $v=$ $1, \ldots, 6$. The two lowest and the two highest bands are distinguished by different line styles. The inset in (a) shows the eigenenergy for $M=8$ and $h=1.25 \AA$, which gives $c=10 \AA$ and $R \approx 3.419 \AA$.

by $p_{y}$ orbitals and narrower $\pi$ bands formed by $p_{z}$ and $p_{x}$ orbitals. When the SOI is considered in the straight chain, four $\pi$ bands of $\left(p_{z}, \sigma= \pm 1\right)$ and $\left(p_{x}, \sigma= \pm 1\right)$ split into two doubly spin degenerate bands, and band anticrossings appear due to the mixing between $\sigma$ and $\pi$ bands. In helical chains the hopping between different orbitals additionally contributes to both the band splitting and anticrossing. The inset in Fig. 2(a) shows the band structure of a helical chain with small $R$ in which the band splitting and anticrossing are prominent, leading to a complicated band structure. In order to obtain a simple picture for the dependence of the spin selectivity on the geometry of the helix, we restrict most of our discussion to the $\sigma$-like bands in a helical chain with large $R$, which are simple parabolic bands, as shown in Fig. 2(a). With respect to the $\pi$-like bands, we mention only that four $\pi$-like bands in the vicinity of $\kappa=0$ in Fig. 2(a) correspond to the four $\pi$-like bands in an analytical continuum model of a helical tube [82]: the major difference originates from the difference in magnitude of the parameters; the $L S$ coupling of iodine produces the largest energy splitting in the present calculation.

Figures 2(b) and 2(c) show eigenstate expectation values of orbital and spin angular momenta, $\left\langle\nu \kappa\left|\hat{L}_{Z}\right| v \kappa\right\rangle$ and $\left\langle\nu \kappa\left|\hat{\sigma}_{Z}\right| \nu \kappa\right\rangle$, respectively, as a function of $\kappa$ for $v=1, \ldots, 6$. Expectation values of $X$ and $Y$ components vanish for both
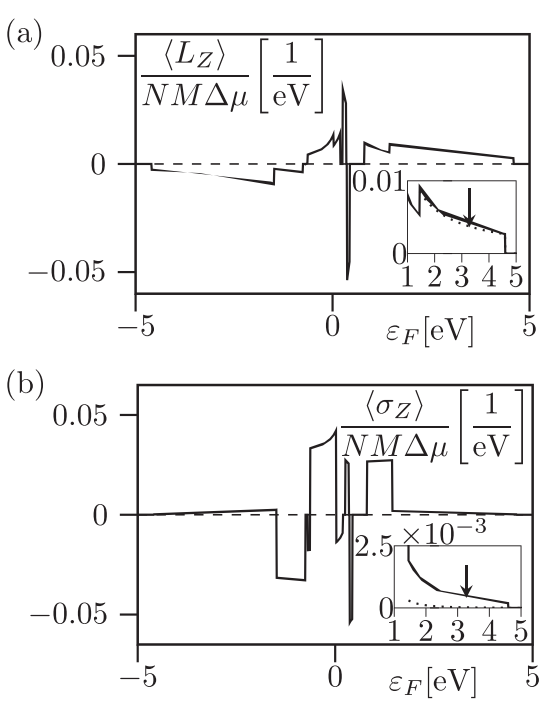

FIG. 3. (a) Current-induced polarization of the orbital angular momentum $\left\langle L_{Z}\right\rangle / N M \Delta \mu$ and (b) that of the spin angular momentum $\left\langle\sigma_{Z}\right\rangle / N M \Delta \mu$ as a function of the Fermi energy $\varepsilon_{F}$ in a helical atomic chain having $M=100$ and $h=0.1 \AA$, which gives $c=10 \AA$ and $R \approx 46.14 \AA$. The current direction is $+Z$. Insets show a magnified plot, with the dotted line indicating the contribution from the difference in $\left|\left\langle v_{\kappa} \kappa\left|\hat{L}_{Z}\right| v_{\kappa} \kappa\right\rangle\right|$ [Eq. (12)] and $\left|\left\langle v_{\kappa} \kappa\left|\hat{\sigma}_{Z}\right| v_{\kappa} \kappa\right\rangle\right|$ [Eq. (13)]. Arrows indicate the value of the Fermi energy $\left(\varepsilon_{F}=3.275 \mathrm{eV}\right)$ when the number of holes is 0.5 per atom.

orbital and spin angular momenta due to the symmetry. The $Z$ component varies significantly in the $\kappa$ region where band anticrossings occur. On the other hand, within the $\sigma$-like bands, $\left\langle\nu \kappa\left|\hat{\sigma}_{Z}\right| \nu \kappa\right\rangle$ takes a value close to either $\sigma=1$ or $\sigma=-1$, while $\left\langle\nu \kappa\left|\hat{L}_{Z}\right| \nu \kappa\right\rangle$ gradually changes with $\kappa$. In accordance with the time reversal symmetry, each eigenstate at $\kappa$ in Fig. 2 has a partner eigenstate of the same energy at $-\kappa$ whose expectation values of orbital and spin angular momenta are $-\left\langle\nu \kappa\left|\hat{L}_{Z}\right| \nu \kappa\right\rangle$ and $-\left\langle\nu \kappa\left|\hat{\sigma}_{Z}\right| \nu \kappa\right\rangle$, respectively.

\section{Current-induced polarizations of orbital and spin angular momenta}

\section{Fermi-energy dependence}

Figure 3 shows the dependence on the Fermi energy $\varepsilon_{F}$ of current-induced polarizations, $\left\langle L_{Z}\right\rangle$ and $\left\langle\sigma_{Z}\right\rangle$. Discontinuous changes as a function of $\varepsilon_{F}$ appear at the opening of an additional channel at which the density of states diverges. Within the $\sigma$-like bands, on the other hand, both $\left\langle L_{Z}\right\rangle$ and $\left\langle\sigma_{Z}\right\rangle$ gradually change with $\varepsilon_{F}$. In this $\varepsilon_{F}$ region, two channels are present which give polarizations of the opposite direction. The sum of contributions from the two channels to each of $\left\langle L_{Z}\right\rangle$ [Eq. (12)] and $\left\langle\sigma_{Z}\right\rangle$ [Eq. (13)] acquires a nonzero value owing to the difference between the two channels in $\left|\left\langle v_{\kappa} \kappa\left|\hat{L}_{Z}\right| v_{\kappa} \kappa\right\rangle\right|$ and $\left|\left\langle v_{\kappa} \kappa\left|\hat{\sigma}_{Z}\right| v_{\kappa} \kappa\right\rangle\right|$ and that in the density of states $\rho_{\kappa}$. We divide $\left\langle L_{Z}\right\rangle$ and $\left\langle\sigma_{Z}\right\rangle$ into two terms, each of which originates from one of these differences,

$$
\begin{aligned}
\frac{\left\langle L_{Z}\right\rangle}{N c \Delta \mu} & =L_{\uparrow} \rho_{\uparrow}+L_{\downarrow} \rho_{\downarrow} \\
& =\left(L_{\uparrow}+L_{\downarrow}\right) \frac{\rho_{\uparrow}+\rho_{\downarrow}}{2}+\frac{L_{\uparrow}-L_{\downarrow}}{2}\left(\rho_{\uparrow}-\rho_{\downarrow}\right),
\end{aligned}
$$



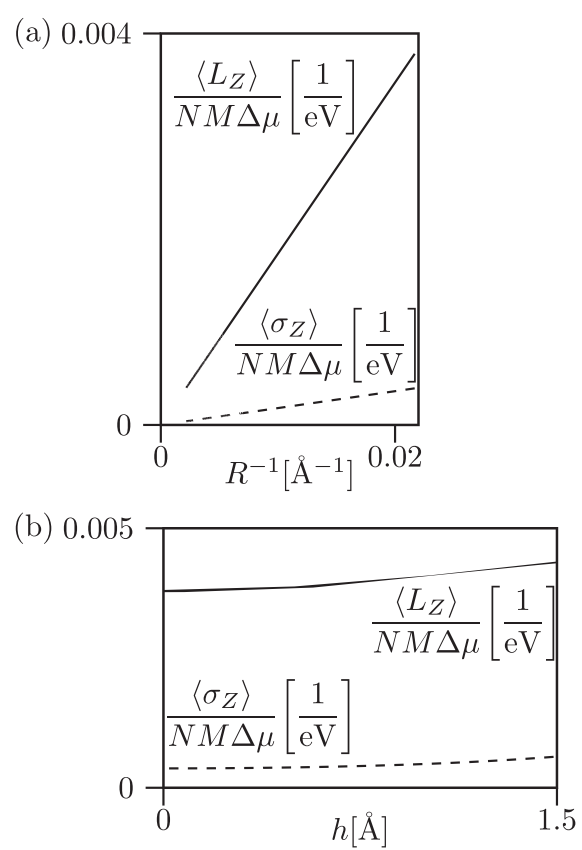

FIG. 4. (a) Current-induced polarization of the orbital angular momentum $\left\langle L_{Z}\right\rangle / N M \Delta \mu$ and that of the spin angular momentum $\left\langle\sigma_{Z}\right\rangle / N M \Delta \mu$ as a function of $R^{-1}$ in a helical atomic chain having $h=0.1 \AA$. The number of holes is fixed to 0.5 per atom. Since $c=M h, 2 R \sin (\pi / M)=\sqrt{d^{2}-h^{2}}$, and $M$ is changed from 100 to 2000, $c$ increases from 10 to $200 \AA$, and $R$ increases from 46.14 to $922.6 \AA$. The current direction is $+Z$. (b) The same as (a), plotted as a function of $h$ in a helical atomic chain having $M=100$. With $h$ increasing from 0 to $1.5 \AA, c$ increases from 0 to $150 \AA$, and $R$ decreases from 46.16 to $39.51 \AA$.

$$
\begin{aligned}
\frac{\left\langle\sigma_{Z}\right\rangle}{N c \Delta \mu} & =\sigma_{\uparrow} \rho_{\uparrow}+\sigma_{\downarrow} \rho_{\downarrow} \\
& =\left(\sigma_{\uparrow}+\sigma_{\downarrow}\right) \frac{\rho_{\uparrow}+\rho_{\downarrow}}{2}+\frac{\sigma_{\uparrow}-\sigma_{\downarrow}}{2}\left(\rho_{\uparrow}-\rho_{\downarrow}\right),
\end{aligned}
$$

where $L_{\uparrow}, \sigma_{\uparrow}$, and $\rho_{\uparrow}$ are $\left\langle v_{\kappa} \kappa\left|\hat{L}_{Z}\right| v_{\kappa} \kappa\right\rangle,\left\langle v_{\kappa} \kappa\left|\hat{\sigma}_{Z}\right| v_{\kappa} \kappa\right\rangle$, and $\rho_{\kappa}$ of the "spin-up" channel with $\left\langle v_{\kappa} \kappa\left|\hat{\sigma}_{Z}\right| v_{\kappa} \kappa\right\rangle \approx 1$ and $L_{\downarrow}, \sigma_{\downarrow}$, and $\rho_{\downarrow}$ are those of the "spin-down" channel. As indicated by dotted lines in the insets in Figs. 3(a) and 3(b), the term due to the difference in $\left|\left\langle v_{\kappa} \kappa\left|\hat{L}_{Z}\right| v_{\kappa} \kappa\right\rangle\right|$ is dominant in $\left\langle L_{Z}\right\rangle$, while that due to the difference in the density of states is dominant in $\left\langle\sigma_{Z}\right\rangle$. We emphasize that the channel dependence of the angular momentum expectation value $\left|\left\langle v_{\kappa} \kappa\left|\hat{L}_{Z}\right| v_{\kappa} \kappa\right\rangle\right|$ [Fig. 2(b)] can be described only by taking into account the intra-atomic SOI. Such dependence gives rise to the CIOP $\left\langle L_{Z}\right\rangle$, which acts as an effective magnetic field to produce the $\operatorname{CISP}\left\langle\sigma_{Z}\right\rangle$.

\section{Geometry dependence}

Figures 4(a) and 4(b) present the dependence on the radius $R$ and that on the pitch $c=M h$, respectively, of currentinduced polarizations, $\left\langle L_{Z}\right\rangle$ and $\left\langle\sigma_{Z}\right\rangle$. The change in $R$ and $c$ gives rise to the change in the band structure, which can vary the number of channels when the Fermi energy is fixed. Therefore, we fix the number of electrons (holes) instead. In Fig. 4 we choose the value of 5.5 electrons $(0.5$ hole $)$ per atom, at which the Fermi level is in the $\sigma$-like bands, as shown in the insets in Fig. 3.

It is found in Fig. 4(a) that both $\left\langle L_{Z}\right\rangle$ and $\left\langle\sigma_{Z}\right\rangle$ are proportional to $1 / R$, the curvature of the cylinder surface (Fig. 1), in the small curvature region: $\left\langle L_{Z}\right\rangle=A_{L} R^{-1}$ and $\left\langle\sigma_{Z}\right\rangle=A_{S} R^{-1}$, with constants $A_{L}$ and $A_{S}$. The reason is that at $R^{-1} \rightarrow 0$ (straight chain), the inversion symmetry is restored, leading to the vanishing of $\left\langle L_{Z}\right\rangle$ and $\left\langle\sigma_{Z}\right\rangle$. The proportionality in $\left\langle L_{Z}\right\rangle$ suggests that the orbital angular momentum in each atom couples to the current through a helical atomic chain with strength proportional to the curvature. The proportionality in $\left\langle\sigma_{Z}\right\rangle$ is derived from that in $\left\langle L_{Z}\right\rangle$ since the orbital angular momentum polarization acts as an effective magnetic field on the spin through the $L S$ coupling. Based on this mechanism, we deduce that enhancing the orbital angular momentum polarization through the curvature will be effective in obtaining a large spin polarization.

Figure 4(b) indicates that $\left\langle L_{Z}\right\rangle=B_{L}+D_{L} h^{2}$ and $\left\langle\sigma_{Z}\right\rangle=$ $B_{S}+D_{S} h^{2}$ in the small $h$ region with constants $B_{L}, D_{L}, B_{S}$, and $D_{S}$. Linear-in- $h$ terms are absent because $\left\langle L_{Z}\right\rangle$ and $\left\langle\sigma_{Z}\right\rangle$ are even functions of $h$. Importantly, both $\left\langle L_{Z}\right\rangle$ and $\left\langle\sigma_{Z}\right\rangle$ have a nonzero value at $c=0(h=0)$. The structure with $c<d$ including $c=0$ ( $d$ is the interatomic distance along the helical curve) is beyond the application range of this model since the electron hopping in the $Z$ direction is neglected here (see Fig. 1). Nevertheless, the result at $c=0$ can be useful in investigating the origin of the CISP. In order to consider only the hopping along the chain, consider a circular arc in a plane instead of a helix in the limit of $c=0$. The circular arc has a curvature and no chirality. Nonzero CISP at $c=0$ in Fig. 4(b) combined with the result in Fig. 4(a), which shows the curvature produces the CISP, suggests the possibility that an atomic chain along a circular arc can produce the CISP.

With this possibility of the CISP in nonchiral structures with curvature in mind, we propose to understand the existence of the CISP in the present model by the chirality in the current, which is one of the fundamental concepts in the quantum Hall effect and other topological phenomena [83,84]. Here we define the chirality in the current by the direction of the current flow when viewed from the positive $Z$ direction: the chirality in the current is, say, positive (negative) for the counterclockwise (clockwise) current flow. Then the chirality in the current is positive for both $c>0$ and $c=0$ in Fig. 4(b), which is consistent with the existence of $\left\langle\sigma_{Z}\right\rangle$ at $c=0$.

It is also shown in our calculation that helical atomic chains with opposite chiralities in the structure have $\left\langle\sigma_{Z}\right\rangle$ with opposite signs for the current in a fixed direction along the helix axis, say, in the positive $Z$ direction $(I>0)$ [8-10,22,23,26,27]: if $\left\langle\sigma_{Z} \gg 0\right.$ for positive chirality in structure, then $\left\langle\sigma_{Z}\right\rangle<0$ for negative chirality. Of course, the sign of $\left\langle\sigma_{Z}\right\rangle$ is reversed for $I<0$. Such dependences of $\left\langle\sigma_{Z}\right\rangle$ on the sign of $I$ and on the chirality in the structure can be summarized as the dependence of $\left\langle\sigma_{Z}\right\rangle$ on the chirality in the current.

\section{CONCLUSIONS}

We have theoretically studied current-induced spin and orbital polarizations in a helical atomic chain with $p_{x}, p_{y}$, and $p_{z}$ orbitals in each atom by calculating polarizations of 
the spin and atomic orbital angular momenta in the linear response to the chemical-potential difference between source and drain electrodes. We have shown that these polarizations are parallel to the helix axis and proportional to the curvature of the cylinder surface defined by the helix in the small curvature region. This proportionality suggests that the curvature generates the coupling between the current and the orbital angular momentum and therefore influences the magnitude of induced spin polarization through the $L S$ coupling. With respect to the eigenstate, it has been stated in the theory of the SOI in carbon nanotubes [85] that, because of a nonzero curvature of the nanotube, the eigenstate expectation value of the atomic orbital angular momentum is induced, which acts on the spin as an effective magnetic field.

We have also found that current-induced polarizations are nonzero even when the pitch is forced to vanish, which suggests the possibility that nonchiral structures with curvature can produce current-induced polarizations. With this possibility in mind, we have proposed that the chirality in the current, rather than that in the structure, determines the existence of such current-induced polarizations.
Finally, we compare the present study to a closely related previous theory of the spin and orbital magnetization induced by current in a crystal with helical structure [64]. The model of the helical structure in the previous study [64] considers a single orbital in each site and the SOI in intersite hopping integrals, while the present model has the SOI within each atom as the $L S$ coupling. The orbital magnetization calculated in the previous study [64] is due to the electron motion in the crystal structure, while the present study has considered the orbital angular momentum within each atom, which plays the role of the effective magnetic field acting on the spin through the $L S$ coupling. In spite of these differences, the directions of the spin and orbital magnetizations in the previous study [64] and those of the spin and orbital polarizations in the present study are both parallel to the current which flows along the helix axis.

\section{ACKNOWLEDGMENTS}

This work was supported by Grant-in-Aid for Scientific Research (C) Grant No. JP17K05484 from the Japan Society for the Promotion of Science (JSPS).
[1] I. Žutić, J. Fabian, and S. D. Sarma, Spintronics: Fundamentals and applications, Rev. Mod. Phys. 76, 323 (2004).

[2] F. Evers, R. Korytár, S. Tewari, and J. M. van Ruitenbeek, Advances and challenges in single-molecule electron transport, Rev. Mod. Phys. 92, 035001 (2020).

[3] S. G. Ray, S. S. Daube, G. Leitus, Z. Vager, and R. Naaman, Chirality-Induced Spin-Selective Properties of Self-Assembled Monolayers of DNA on Gold, Phys. Rev. Lett. 96, 036101 (2006)

[4] B. Göhler, V. Hamelbeck, T. Z. Markus, M. Kettner, G. F. Hanne, Z. Vager, R. Naaman, and H. Zacharias, Spin selectivity in electron transmission through self-assembled monolayers of double-stranded DNA, Science 331, 894 (2011).

[5] Z. Xie, T. Z. Markus, S. R. Cohen, Z. Vager, R. Gutierrez, and R. Naaman, Spin specific electron conduction through DNA oligomers, Nano Lett. 11, 4652 (2011).

[6] D. Mishra, T. Z. Markus, R. Naaman, M. Kettner, B. Göhler, H. Zacharias, N. Friedman, M. Sheves, and C. Fontanesi, Spindependent electron transmission through bacteriorhodopsin embedded in purple membrane, Proc. Natl. Acad. Sci. USA 110, 14872 (2013).

[7] M. Kettner, B. Göhler, H. Zacharias, D. Mishra, V. Kiran, R. Naaman, C. Fontanesi, D. H. Waldeck, S. Sek, J. Pawłowski, and J. Juhaniewicz, Spin filtering in electron transport through chiral oligopeptides, J. Phys. Chem. C 119, 14542 (2015).

[8] V. Kiran, S. P. Mathew, S. R. Cohen, I. Hernández Delgado, J. Lacour, and R. Naaman, Helicenes-A new class of organic spin filter, Adv. Mater. 28, 1957 (2016).

[9] A. C. Aragonès, E. Medina, M. Ferrer-Huerta, N. Gimeno, M. Teixidó, J. L. Palma, N. Tao, J. M. Ugalde, E. Giralt, I. DíezPérez, and V. Mujica, Measuring the spin-polarization power of a single chiral molecule, Small 13, 1602519 (2017).
[10] M. Kettner, V. V. Maslyuk, D. Nürenberg, J. Seibel, R. Gutierrez, G. Cuniberti, K.-H. Ernst, and H. Zacharias, Chirality-dependent electron spin filtering by molecular monolayers of helicenes, J. Phys. Chem. Lett. 9, 2025 (2018).

[11] H. Lu, J. Wang, C. Xiao, X. Pan, X. Chen, R. Brunecky, J. J. Berry, K. Zhu, M. C. Beard, and Z. V. Vardeny, Spin-dependent charge transport through 2D chiral hybrid lead-iodide perovskites, Sci. Adv. 5, eaay0571 (2019).

[12] S. Mishra, A. K. Mondal, S. Pal, T. K. Das, E. Z. B. Smolinsky, G. Siligardi, and R. Naaman, Length-dependent electron spin polarization in oligopeptides and DNA, J. Phys. Chem. C 124, 10776 (2020).

[13] T. Liu, X. Wang, H. Wang, G. Shi, F. Gao, H. Feng, H. Deng, L. Hu, E. Lochner, P. Schlottmann, S. von Molnár, Y. Li, J. Zhao, and P. Xiong, Linear and nonlinear two-terminal spinvalve effect from chirality-induced spin selectivity, ACS Nano 14, 15983 (2020).

[14] C. Kulkarni, A. K. Mondal, T. K. Das, G. Grinbom, F. Tassinari, M. F. J. Mabesoone, E. W. Meijer, and R. Naaman, Highly efficient and tunable filtering of electrons' spin by supramolecular chirality of nanofiber-based materials, Adv. Mater. 32, 1904965 (2020).

[15] L. Jia, C. Wang, Y. Zhang, L. Yang, and Y. Yan, Efficient spin selectivity in self-assembled superhelical conducting polymer microfibers, ACS Nano 14, 6607 (2020).

[16] R. Naaman and D. H. Waldeck, Spintronics and chirality: Spin selectivity in electron transport through chiral molecules, Annu. Rev. Phys. Chem. 66, 263 (2015).

[17] O. B. Dor, S. Yochelis, S. P. Mathew, R. Naaman, and Y. Paltiel, A chiral-based magnetic memory device without a permanent magnet, Nat. Commun. 4, 2256 (2013).

[18] M. Suda, Y. Thathong, V. Promarak, H. Kojima, M. Nakamura, T. Shiraogawa, M. Ehara, and H. M. Yamamoto, Light-driven 
molecular switch for reconfigurable spin filters, Nat. Commun. 10, 2455 (2019).

[19] V. Kiran, S. R. Cohen, and R. Naaman, Structure dependent spin selectivity in electron transport through oligopeptides, J. Chem. Phys. 146, 092302 (2017).

[20] R. Gutierrez, E. Díaz, R. Naaman, and G. Cuniberti, Spinselective transport through helical molecular systems, Phys. Rev. B 85, 081404(R) (2012).

[21] A.-M. Guo and Q.-F. Sun, Spin-Selective Transport of Electrons in DNA Double Helix, Phys. Rev. Lett. 108, 218102 (2012).

[22] A.-M. Guo and Q.-F. Sun, Spin-dependent electron transport in protein-like single-helical molecules, Proc. Natl. Acad. Sci. USA 111, 11658 (2014).

[23] T.-R. Pan, A.-M. Guo, and Q.-F. Sun, Spin-polarized electron transport through helicene molecular junctions, Phys. Rev. B 94, 235448 (2016).

[24] H.-N. Wu, X. Wang, Y.-J. Zhang, G.-Y. Yi, and W.-J. Gong, Enhancement of spin polarization in transport through protein-like single-helical molecules, Appl. Phys. A 122, 626 (2016).

[25] S. Matityahu, Y. Utsumi, A. Aharony, O. Entin-Wohlman, and C. A. Balseiro, Spin-dependent transport through a chiral molecule in the presence of spin-orbit interaction and nonunitary effects, Phys. Rev. B 93, 075407 (2016).

[26] S. Matityahu, A. Aharony, O. Entin-Wohlman, and C. A. Balseiro, Spin filtering in all-electrical three-terminal interferometers, Phys. Rev. B 95, 085411 (2017).

[27] L.-L. Nian, X.-J. Ge, and L. Bai, Nanoscale spintronics switch based on a three-terminal dsDNA molecule junction, J. Phys. D 52, 105301 (2019).

[28] J. Fransson, Chirality-induced spin selectivity: The role of electron correlations, J. Phys. Chem. Lett. 10, 7126 (2019).

[29] J. Fransson, Vibrational origin of exchange splitting and chiralinduced spin selectivity, Phys. Rev. B 102, 235416 (2020).

[30] P.-J. Hu, S.-X. Wang, X.-H. Gao, Y.-Y. Zhang, T.-F. Fang, A.-M. Guo, and Q.-F. Sun, Spin-dependent electron transport along hairpinlike DNA molecules, Phys. Rev. B 102, 195406 (2020).

[31] M. A. Sierra, D. Sánchez, R. Gutierrez, G. Cuniberti, F. Domínguez-Adame, and E. Díaz, Spin-polarized electron transmission in DNA-like systems, Biomolecules 10, 49 (2020).

[32] L. Zhang, Y. Hao, W. Qin, S. Xie, and F. Qu, Chiral-induced spin selectivity: A polaron transport model, Phys. Rev. B 102, 214303 (2020).

[33] S. Varela, V. Mujica, and E. Medina, Effective spin-orbit couplings in an analytical tight-binding model of DNA: Spin filtering and chiral spin transport, Phys. Rev. B 93, 155436 (2016).

[34] S. Varela, V. Mujica, and E. Medina, Spin-orbit coupling modulation in DNA by mechanical deformations, Chimia 72, 411 (2018).

[35] S. Varela, B. Montañes, F. López, B. Berche, B. Guillot, V. Mujica, and E. Medina, Intrinsic Rashba coupling due to hydrogen bonding in DNA, J. Chem. Phys. 151, 125102 (2019).

[36] M. Geyer, R. Gutierrez, V. Mujica, and G. Cuniberti, Chiralityinduced spin selectivity in a coarse-grained tight-binding model for helicene, J. Phys. Chem. C 123, 27230 (2019).

[37] J. D. Torres, R. Hidalgo-Sacoto, S. Varela, and E. Medina, Mechanically modulated spin-orbit couplings in oligopeptides, Phys. Rev. B 102, 035426 (2020).
[38] Y. Utsumi, O. Entin-Wohlman, and A. Aharony, Spin selectivity through time-reversal symmetric helical junctions, Phys. Rev. B 102, 035445 (2020).

[39] X. Li, J. Nan, and X. Pan, Chiral Induced Spin Selectivity as a Spontaneous Intertwined Order, Phys. Rev. Lett. 125, 263002 (2020).

[40] V. V. Maslyuk, R. Gutierrez, A. Dianat, V. Mujica, and G. Cuniberti, Enhanced magnetoresistance in chiral molecular junctions, J. Phys. Chem. Lett. 9, 5453 (2018).

[41] Y. Matsuura, Coherent spin transport in a DNA molecule, Chem. Phys. 528, 110537 (2020).

[42] Y. Matsuura and F. Kato, Structure-dependent spin transport in a DNA molecule, Comput. Theor. Chem. 1188, 112943 (2020).

[43] Y. Matsuura and F. Kato, Spin transport in a DNA molecule modified by cytosine methylation, Chem. Phys. 539, 110946 (2020).

[44] M. S. Zöllner, S. Varela, E. Medina, V. Mujica, and C. Herrmann, Insight into the origin of chiral-induced spin selectivity from a symmetry analysis of electronic transmission, J. Chem. Theory Comput. 16, 2914 (2020).

[45] M. S. Zöllner, A. Saghatchi, V. Mujica, and C. Herrmann, Influence of electronic structure modeling and junction structure on first-principles chiral induced spin selectivity, J. Chem. Theory Comput. 16, 7357 (2020).

[46] S. Dalum and P. Hedegård, Theory of chiral induced spin selectivity, Nano Lett. 19, 5253 (2019).

[47] X. Fan, E. C. Dickey, P. C. Eklund, K. A. Williams, L. Grigorian, R. Buczko, S. T. Pantelides, and S. J. Pennycook, Atomic Arrangement of Iodine Atoms Inside Single-Walled Carbon Nanotubes, Phys. Rev. Lett. 84, 4621 (2000).

[48] L. Guan, K. Suenaga, Z. Shi, Z. Gu, and S. Iijima, Polymorphic structures of iodine and their phase transition in confined nanospace, Nano Lett. 7, 1532 (2007).

[49] T. Fujimori, R. B. dos Santos, T. Hayashi, M. Endo, K. Kaneko, and D. Tománek, Formation and properties of selenium doublehelices inside double-wall carbon nanotubes: Experiment and theory, ACS Nano 7, 5607 (2013).

[50] P. V. C. Medeiros, S. Marks, J. M. Wynn, A. Vasylenko, Q. M. Ramasse, D. Quigley, J. Sloan, and A. J. Morris, Single-atom scale structural selectivity in Te nanowires encapsulated inside ultranarrow, single-walled carbon nanotubes, ACS Nano 11, 6178 (2017).

[51] J.-K. Qin, P.-Y. Liao, M. Si, S. Gao, G. Qiu, J. Jian, Q. Wang, S.-Q. Zhang, S. Huang, A. Charnas, Y. Wang, M. J. Kim, W. Wu, X. Xu, H.-Y. Wang, L. Yang, Y. K. Yap, and P. D. Ye, Raman response and transport properties of tellurium atomic chains encapsulated in nanotubes, Nat. Electron. 3, 141 (2020).

[52] M. Springborg and Y. Dong, Elemental chains, Int. J. Quantum Chem. 109, 837 (2009).

[53] D. Rybkovskiy, A. Osadchy, and E. Obraztsova, Electronic band structure of helical iodine chains, Phys. Status Solidi B 249, 2608 (2012).

[54] G. S. Diniz, A. Latgé, and S. E. Ulloa, Helicoidal Fields and Spin Polarized Currents in Carbon Nanotube-DNA Hybrids, Phys. Rev. Lett. 108, 126601 (2012).

[55] K. M. Alam and S. Pramanik, Spin filtering through single-wall carbon nanotubes functionalized with single-stranded DNA, Adv. Funct. Mater. 25, 3210 (2015). 
[56] Y. Perlitz and K. Michaeli, Helical liquid in carbon nanotubes wrapped with DNA molecules, Phys. Rev. B 98, 195405 (2018)

[57] A. G. Aronov and Y. B. Lyanda-Geller, Nuclear electric resonance and orientation of carrier spins by an electric field, JETP Lett. 50, 431 (1989).

[58] V. Edelstein, Spin polarization of conduction electrons induced by electric current in two-dimensional asymmetric electron systems, Solid State Commun. 73, 233 (1990).

[59] M. Trushin and J. Schliemann, Anisotropic current-induced spin accumulation in the two-dimensional electron gas with spin-orbit coupling, Phys. Rev. B 75, 155323 (2007).

[60] V. K. Kalevich and V. L. Korenev, Effect of electric field on the optical orientation of 2D electrons, JETP Lett. 52, 230 (1990).

[61] A. Y. Silov, P. A. Blajnov, J. H. Wolter, R. Hey, K. H. Ploog, and N. S. Averkiev, Current-induced spin polarization at a single heterojunction, Appl. Phys. Lett. 85, 5929 (2004).

[62] Y. K. Kato, R. C. Myers, A. C. Gossard, and D. D. Awschalom, Current-Induced Spin Polarization in Strained Semiconductors, Phys. Rev. Lett. 93, 176601 (2004).

[63] C. L. Yang, H. T. He, L. Ding, L. J. Cui, Y. P. Zeng, J. N. Wang, and W. K. Ge, Spectral Dependence of Spin Photocurrent and Current-Induced Spin Polarization in an InGaAs/InAlAs Two-Dimensional Electron Gas, Phys. Rev. Lett. 96, 186605 (2006).

[64] T. Yoda, T. Yokoyama, and S. Murakami, Current-induced orbital and spin magnetizations in crystals with helical structure, Sci. Rep. 5, 12024 (2015).

[65] A. Shitade and E. Minamitani, Geometric spin-orbit coupling and chirality-induced spin selectivity, New J. Phys. 22, 113023 (2020).

[66] A. R. Mellnik, J. S. Lee, A. Richardella, J. L. Grab, P. J. Mintun, M. H. Fischer, A. Vaezi, A. Manchon, E.-A. Kim, N. Samarth, and D. C. Ralph, Spin-transfer torque generated by a topological insulator, Nature (London) 511, 449 (2014).

[67] M. B. Jungfleisch, W. Zhang, J. Sklenar, W. Jiang, J. E. Pearson, J. B. Ketterson, and A. Hoffmann, Interface-driven spin-torque ferromagnetic resonance by Rashba coupling at the interface between nonmagnetic materials, Phys. Rev. B 93, 224419 (2016).

[68] K. Kondou, R. Yoshimi, A. Tsukazaki, Y. Fukuma, J. Matsuno, K. S. Takahashi, M. Kawasaki, Y. Tokura, and Y. Otani, Fermilevel-dependent charge-to-spin current conversion by Dirac surface states of topological insulators, Nat. Phys. 12, 1027 (2016).

[69] A. Inui, R. Aoki, Y. Nishiue, K. Shiota, Y. Kousaka, H. Shishido, D. Hirobe, M. Suda, J.-I. Ohe, J.-I. Kishine, H. M. Yamamoto, and Y. Togawa, Chirality-Induced Spin-Polarized State of a Chiral Crystal $\mathrm{CrNb}_{3} \mathrm{~S}_{6}$, Phys. Rev. Lett. 124, 166602 (2020).
[70] J. H. Ryoo and C.-H. Park, Hidden orbital polarization in diamond, silicon, germanium, gallium arsenide and layered materials, NPG Asia Mater. 9, e382 (2017).

[71] D. Go, J.-P. Hanke, P. M. Buhl, F. Freimuth, G. Bihlmayer, H.-W. Lee, Y. Mokrousov, and S. Blügel, Toward surface orbitronics: Giant orbital magnetism from the orbital Rashba effect at the surface of $s p$-metals, Sci. Rep. 7, 46742 (2017).

[72] X. Chen, Y. Liu, G. Yang, H. Shi, C. Hu, M. Li, and H. Zeng, Giant antidamping orbital torque originating from the orbital Rashba-Edelstein effect in ferromagnetic heterostructures, Nat. Commun. 9, 2569 (2018).

[73] L. Salemi, M. Berritta, A. K. Nandy, and P. M. Oppeneer, Orbitally dominated Rashba-Edelstein effect in noncentrosymmetric antiferromagnets, Nat. Commun. 10, 5381 (2019).

[74] B. A. Bernevig, T. L. Hughes, and S.-C. Zhang, Orbitronics: The Intrinsic Orbital Current in $p$-Doped Silicon, Phys. Rev. Lett. 95, 066601 (2005).

[75] H. Kontani, T. Tanaka, D. S. Hirashima, K. Yamada, and J. Inoue, Giant Intrinsic Spin and Orbital Hall Effects in $\mathrm{Sr}_{2} \mathrm{MO}_{4}$ ( $M=\mathrm{Ru}, \mathrm{Rh}, \mathrm{Mo}$ ), Phys. Rev. Lett. 100, 096601 (2008).

[76] H. Kontani, T. Tanaka, D. S. Hirashima, K. Yamada, and J. Inoue, Giant Orbital Hall Effect in Transition Metals: Origin of Large Spin and Anomalous Hall Effects, Phys. Rev. Lett. 102, 016601 (2009).

[77] D. Go, D. Jo, C. Kim, and H.-W. Lee, Intrinsic Spin and Orbital Hall Effects from Orbital Texture, Phys. Rev. Lett. 121, 086602 (2018)

[78] Y. Liu, J. Xiao, J. Koo, and B. Yan, Chirality-driven topological electronic structure of DNA-like materials, Nat. Mater. 20, 638 (2021).

[79] J. C. Slater and G. F. Koster, Simplified LCAO method for the periodic potential problem, Phys. Rev. 94, 1498 (1954).

[80] W. Izumida, K. Sato, and R. Saito, Spin-orbit interaction in single wall carbon nanotubes: Symmetry adapted tight-binding calculation and effective model analysis, J. Phys. Soc. Jpn. 78 , 074707 (2009).

[81] P. Carrier and S.-H. Wei, Calculated spin-orbit splitting of all diamondlike and zinc-blende semiconductors: Effects of $p_{1 / 2}$ local orbitals and chemical trends, Phys. Rev. B 70, 035212 (2004).

[82] K. Michaeli and R. Naaman, Origin of spin-dependent tunneling through chiral molecules, J. Phys. Chem. C 123, 17043 (2019).

[83] B. I. Halperin, Quantized Hall conductance, current-carrying edge states, and the existence of extended states in a twodimensional disordered potential, Phys. Rev. B 25, 2185 (1982).

[84] M. Z. Hasan and C. L. Kane, Colloquium: Topological insulators, Rev. Mod. Phys. 82, 3045 (2010).

[85] T. Ando, Spin-orbit interaction in carbon nanotubes, J. Phys. Soc. Jpn. 69, 1757 (2000). 